\title{
Genetic diversity and origin of North American green foxtail [Setaria viridis (L.) Beauv.] accessions
}

\author{
Stephan Schröder • Bochra A. Bahri • Douglas M. Eudy • \\ Daniel J. Layton • Elizabeth A. Kellogg • Katrien M. Devos
}

Received: 16 July 2015/Accepted: 11 January 2016/Published online: 25 January 2016

(C) The Author(s) 2016. This article is published with open access at Springerlink.com

\begin{abstract}
Setaria viridis (L.) P. Beauv. and its domesticated form, S. italica (L.) P. Beauv., have been developed over the past few years as model systems for $\mathrm{C}_{4}$ photosynthesis and for the analysis of bioenergy traits. S. viridis is native to Eurasia, but is now a ubiquitous weed. An analysis of the population structure of a set of $232 \mathrm{~S}$. viridis lines, mostly from North America but also comprising some accessions from around the world, using 11 SSR markers, showed that $S$. viridis populations in the US largely separate by latitude and/or climatic zone. S. viridis populations from the Northern US and Canada (north of $44^{\circ} \mathrm{N}$ ) group with accessions from Western Europe, while populations in the Mid and Southern US
\end{abstract}

Electronic supplementary material The online version of this article (doi:10.1007/s10722-016-0363-6) contains supplementary material, which is available to authorized users.

S. Schröder · B. A. Bahri · D. M. Eudy .

K. M. Devos $(\bowtie)$

Department of Crop and Soil Sciences, Institute of Plant

Breeding, Genetics and Genomics, University of Georgia,

Athens, GA 30602, USA

e-mail: kdevos@uga.edu

S. Schröder · B. A. Bahri · K. M. Devos

Department of Plant Biology, University of Georgia,

Athens, GA 30602, USA

Present Address:

S. Schröder

Department of Plant Sciences, North Dakota State University, Fargo, ND 58108, USA predominantly group with accessions from Turkey and Iran. We hypothesize that $S$. viridis in the US was most likely introduced from Europe, and that introductions were competitive only in regions that had climatic conditions that were similar to those in the regions of origins. This hypothesis is supported by the fact that Canadian $S$. viridis lines were fast cycling and undersized when grown in the Mid-Western and Southern US compared to their morphology in their native environment. A comparison of the population structure obtained with 11 SSR markers and $\sim 40,000$ single nucleotide polymorphisms (SNPs) in a common set of $S$. viridis germplasm showed that both methods essentially yielded the same groupings, although admixture was identified at a higher frequency in the SNP analysis. Small numbers of SSR markers can thus

\section{B. A. Bahri}

Department of Plant Protection and Postharvest Diseases,

National Agronomic Institute of Tunisia, 1082 Tunis,

Tunisia

D. J. Layton · E. A. Kellogg

Department of Biology, University of Missouri-St. Louis,

St. Louis, MO 63121, USA

Present Address:

E. A. Kellogg

Donald Danforth Plant Science Center, St. Louis, MO 63132, USA 
be used effectively to discern the population structure in this inbreeding species.

Keywords Adaptation - Genetic diversity · Green foxtail [Setaria viridis (L.) Beauv.] · Population structure $\cdot$ Simple sequence repeat

\section{Introduction}

Setaria viridis (L.) Beauv., green foxtail, belongs to the family Poaceae, subfamily Panicoideae, tribe Paniceae. It is the wild progenitor of foxtail millet [S. italica (L.) Beauv.], a crop cultivated for food mainly in China, India and Russia, for birdseed in Europe and for hay and silage in the United States. $S$. viridis can cross freely with the cultivated $S$. italica, and this has been exploited to transfer traits from $S$. viridis to $S$. italica including herbicide resistance (Naciri et al. 1992; Wang et al. 1996). Naciri et al. (1992) reported that only few backcrosses were needed to eliminate weediness following an interspecific cross between $S$. viridis and $S$. italica, making trait introgression from the weed to the cultivated species a viable strategy. S. viridis may also be a source of novel stress tolerance genes for the genetic improvement of S. italica (Qie et al. 2014).

Setaria viridis is an annual diploid species thought to be native to Eurasia (Darmency 2005) but now a ubiquitous weed in temperate regions throughout the world (Invasive Species Compendium: www.cabi.org/ isc/). In North America, S. viridis was first reported in Montreal, Canada in 1821 (Douglas et al. 1985). Most likely it was introduced as a contaminant of crop seed and in the ballast of ships. It remained a relatively minor weed in Canada until at least the 1930s (Manson 1932), but by 1948, the species was widespread throughout Manitoba, Alberta and Saskatchewan (Groh and Frankton 1949). In the US, S. viridis has been present since at least 1900 and has greatly increased in abundance over the past 100 years (Forcella and Harvey 1983). S. viridis is considered one of the most successful plants in colonizing disturbed habitats. It is typically found in agricultural fields, on road sides and along railroad tracks, on ditch banks and in open waste areas. While $S$. viridis is an extensive seed producer, it is a poor competitor and only severely affects crop yields when the seedlings emerge at about the same time as the crop (Blackshaw et al. 1981; Peterson and Nalewaja 1992). Over the past 20 years, green foxtail has acquired resistance to several groups of herbicides (www.weedscience.org) and, as a result, has retained its status as one of top 10 most prevalent weeds in Western Canada.

Setaria viridis is often found in mixed populations with $S$. faberi Herrm. (Japanese bristlegrass) and $S$. pumila (Poir.) Roem. et Schult (yellow foxtail). The first record of S. faberi, giant foxtail, in the US was on Long Island in 1925 (Fairbrothers 1959). It is generally assumed that $S$. faberi was introduced as a seed contaminant from China where the species occurs in several regions as a common weed. It may have spread in the US along the railroads, and became a major agricultural problem in the Corn Belt in the 1950s (Knake 1990). Morphologically, S. faberi is very similar to and easily confused with $S$. viridis (Fairbrothers 1959; Layton and Kellogg 2014). The characteristics that best distinguish the two species are the greater length ratio of the lemma and 2nd glume in $S$. faberi compared to $S$. viridis, and the pubescence on the upper surface of the leaf blades of $S$. faberi. However, leaf pubescence is not universally present in S. faberi in China (Knake 1990). At a genetic level, $S$. faberi is an allotetraploid with one of the genomes being similar to that of the diploid S. viridis. Both species can cross to form triploids ( $\mathrm{Li}$ et al. 1942; Willweber-Kishimoto 1962), and there is thus some potential for gene flow between the two species.

$S$. pumila, yellow foxtail, occurs in multiple ploidy forms $(2 \mathrm{n}=18,36,54$ and 72) (Rominger 1962; chromosome counts database-http://ccdb.tau.ac.il). The species is morphologically and genetically distinct from $S$. viridis. Phylogenetic analysis places $S$. pumila with African Setaria species, suggesting that $S$. pumila is native to Africa (Kellogg et al. 2009). Despite their different genome composition, a recent study reported obtaining seed from a cross between $S$. viridis and S. pumila (Jiang et al. 2013). Although Jiang and colleagues did not investigate the hybrid nature of the seed, and their finding contrasts with earlier reports of unsuccessful attempts to cross both species (Till-Bottraud et al. 1992; Willweber-Kishimoto 1962), we nevertheless need to consider the potential for gene flow between the two species.

Due to its small genome (510 Mb; http://data.kew. org/cvalues/), diploid nature $(2 n=18)$, and short life cycle, $S$. viridis and its domesticated form, $S$. italica, 
have become important models to study the genetics of the biofuel crop switchgrass and for $\mathrm{C}_{4}$ photosynthesis (Brutnell et al. 2010; Doust et al. 2009; Li and Brutnell 2011). The genome of $S$. italica has been sequenced and assembled into 9 pseudomolecules, corresponding to the 9 chromosomes, and covers $\sim 80 \%$ of the genome and more than $95 \%$ of the gene space (Bennetzen et al. 2012; Zhang et al. 2012). The Joint Genome Institute (JGI) has also sequenced several accessions of $S$. viridis using the Illumina platform (sequencing reads available from NCBI's Sequence Read Archive (SRA)).

Several analyses of the diversity of $S$. viridis have been carried out. Wang et al. (1995) analyzed a set of 168 S. viridis accessions, some $75 \%$ of which were collected in North America with the remaining $25 \%$ distributed across Europe and Asia, with 13 isozyme markers. Their results indicated that $S$. viridis had been introduced multiple times from Eurasia into North America and formed two geographically separated subpopulations. Jia et al. (2013) analyzed a set of 288 mainly Chinese accessions with 77 SSR markers and also identified two subpopulations. Although the subpopulations did not correspond to geographical eco-regions, one of the subpopulations comprised mostly accessions from higher latitude eco-regions in Northern China while the majority of lines from lower latitude eco-regions formed the second subpopulation. In our study, we analyzed $S$. viridis and $S$. faberi accessions collected from the US and Canada and compared their DNA profiles obtained with 11 SSR markers with those of a global Setaria collection consisting mainly of $S$. viridis lines but also comprising some $S$. italica accessions.

\section{Materials and methods}

Plant materials

A total of 115 S. viridis accessions (232 lines), $11 S$. italica accessions (11 lines), eight $S$. faberi accessions (22 lines), and one S. verticillata (L.) P. Beauv. (hooked bristlegrass) accession (1 line) were analyzed within this study. The term 'accession' is used in a broad sense and can represent a population of genetically dissimilar individuals growing at the same geographic location, as well as a sample of genetically identical genotypes. Forty-seven S. viridis and seven
S. faberi accessions were collected as part of this project in North America (US and Canada) and for each accession, one to five plants (referred to as 'lines') were sampled per location. A further $12 \mathrm{~S}$. viridis accessions from Canada were obtained from Hugh Beckie, Agriculture and Agri-Food Canada, Saskatoon. In addition, $15 \mathrm{~S}$. viridis accessions from the Middle East, seven from Western Europe, 28 from East Asia, three from South Asia, two from Central America and one from South America were obtained from various sources (Online Resource 1). A list of the accession numbers, species name, country of origin and where known, global positioning system (GPS) coordinates of the collection sites, and source of the seed are given in Online Resource 1.

For the accessions collected in situ as part of this project, single seeds from one to five sampled plants were planted, grown to maturity in the glasshouse under natural day light and selfed. Each of the sampled plants from a single location was given the same accession number with the suffix_1, _2, etc. For accessions obtained from other sources, one or two plants were grown for each accession. Again, these plants were given the same accession number with a number suffix.

\section{Differentiating S. faberi from S. viridis}

S. faberi and S. viridis are morphologically highly similar and largely overlap in their vegetative characters. The main distinguishing characteristics are sparse pubescence on the adaxial side of the leaf blade and a short upper glume not longer than $90 \%$ of the lower lemma length in $S$. faberi compared to glabrous leaves and a glume largely covering the seed in $S$. viridis (Fairbrothers 1959). All lines collected in situ by the authors had been identified as S. viridis or S. faberi prior to the DNA analysis. For the lines obtained from other sources, we used the ratio of the length of the upper glume to lower lemma as the criterion to identify $S$. faberi accessions that had been previously classified as $S$. viridis. Two accessions from China, 8125 and 81-79, had a glume length characteristic of $S$. faberi. Both accessions were considered as $S$. faberi in all analyses.

\section{Genotyping}

DNA was extracted from approximately $10 \mathrm{mg}$ of leaf tissue using a CTAB method (Doyle and Doyle 1987). 
Each line was genotyped with 11 foxtail millet SSRs (p3, p16, p29, p88, p89, p95, b101, b102, b127, b163 and b166; Jia et al. 2009). PCR reactions were carried out in $15 \mu \mathrm{L}$ volumes comprising $50 \mathrm{ng}$ of template DNA, $3 \mu \mathrm{L} 5 \times$ buffer (Promega), $1.5 \mathrm{mM} \mathrm{MgCl}_{2}$, $200 \mu \mathrm{M}$ dNTPs, $67 \mathrm{nM}$ M13-tailed specific forward primer, $267 \mathrm{nM}$ fluorescently labeled M13 primer, $267 \mathrm{nM}$ specific reverse primer and $0.9 \mathrm{U} \mathrm{GoTaq}^{\circledR}$ Flexi DNA Polymerase (Promega). The PCR conditions were $5 \mathrm{~min}$ denaturation at $95^{\circ} \mathrm{C}$, followed by 36 cycles of $15 \mathrm{~s}$ denaturation at $95^{\circ} \mathrm{C}, 30 \mathrm{~s}$ annealing at $57{ }^{\circ} \mathrm{C}$, and $30 \mathrm{~s}$ elongation at $72{ }^{\circ} \mathrm{C}$. A final step of $10 \mathrm{~min}$ at $72{ }^{\circ} \mathrm{C}$ ensured full synthesis of PCR fragments. Three amplicons ( $3 \mu \mathrm{L}$ each) labeled with different fluorochromes were pooled and diluted three-fold. Three $\mu \mathrm{L}$ of the pooled and diluted amplicons were added to $7 \mu \mathrm{L}$ of a 19:1 mix of $\mathrm{Hi}-$ Di formamide (Applied Biosystems) and GeneScan 500 ROX-labeled marker (Applied Biosystems). Amplicons were size-separated on an ABI 3730xl (Applied Biosystems). SSR profiles were evaluated in GeneMarker ${ }^{\circledR}$ (Softgenetics) and peaks were scored manually.

Data analysis

\section{Population structure analysis}

A population structure analysis was conducted using the program STRUCTURE 2.3.1 (Pritchard et al. 2000). No prior information on the geographic origin of the lines was used. Lines that contained identical alleles for all SSRs tested were analyzed as a single entry, so that each entry in the population structure analysis had a different allele composition. Identification of duplicate allele patterns was done using GenAlEx 6.501 (Peakall and Smouse 2012). STRUCTURE was run with K-values varying from 1 to 20 in an admixture model with a burn-in phase of 100,000 iterations and 1,000,000 Markov Chain Monte Carlo (MCMC) iterations and 50 runs for each $\mathrm{K}$. The most likely number of subpopulations (K) was estimated according to Evanno et al. (2005). For a given K, the run with the highest posterior probability (out of 50) was selected for analysis.

Additionally, we analyzed the data with the software InStruct (Gao et al. 2007) which, similarly to STRUCTURE, is a Bayesian clustering method but for inbreeding species. We tested $\mathrm{K}$ values from 2 to 20 with the same parameters as used for STRUCTURE, except that only seven iterations were done for each $\mathrm{K}$.

\section{Principal coordinates and diversity analyses}

All analyses were carried out using GenAlEx version 6.501. The principal coordinates analysis (PCoA) was done with the Covariance-Standardized option (Peakall and Smouse 2012). Correlations between the genetic and the $\log (1+$ geographic distance $)$ transformed geographic distance of samples were analyzed using a Mantel test (Mantel 1967). Analyses of molecular variance (AMOVA) estimated the contribution of each locus to the total variance in each subpopulation and partitioned the total molecular variance within and between subpopulations.

\section{Neighbor-joining tree}

The program MICROSAT v1.5 (Eric Minch, Stanford University, USA; http://hpgl.stanford.edu/projects/ microsat/) was used to calculate genetic distances (Dps) based on the proportion of shared alleles (ps) with Dps $=1-$ ps. The distance matrix was used as input in Phylip v 3.69 to generate a neighbor-joining tree (Felsenstein 1993; http://evolution.genetics. washington.edu/phylip.html). Sample inputs were randomized (J option) and the tree was rooted using $S$. faberi accession 81-79 as outgroup. For comparison, and to check the robustness of the clusters, 12 distance matrices based on shared alleles for (1) all 11 SSR markers and (2) subsets of 10 out of the 11 SSR markers and the corresponding trees were generated using PowerMarker V3.25 (Liu and Muse 2005).

\section{Summary statistics}

GenAlEx version 6.501 (Peakall and Smouse 2012) was used to calculate the number of alleles $(\mathrm{Na})$, effective alleles $(\mathrm{Ne})$ and specific alleles (alleles specific to a single subpopulation) in each subpopulation. In addition, the expected heterozygosity ( $\mathrm{He}$ ) was calculated across the entire population and by subpopulation. Only accessions that (1) were a member of the same subpopulation with a membership $\geq 95 \%$ using both STRUCTURE and InStruct at $\mathrm{K}=3$ and (2) retained their membership to the same overarching population group when $\mathrm{K}$ was increased from 3 to 6 were included in the calculations. 

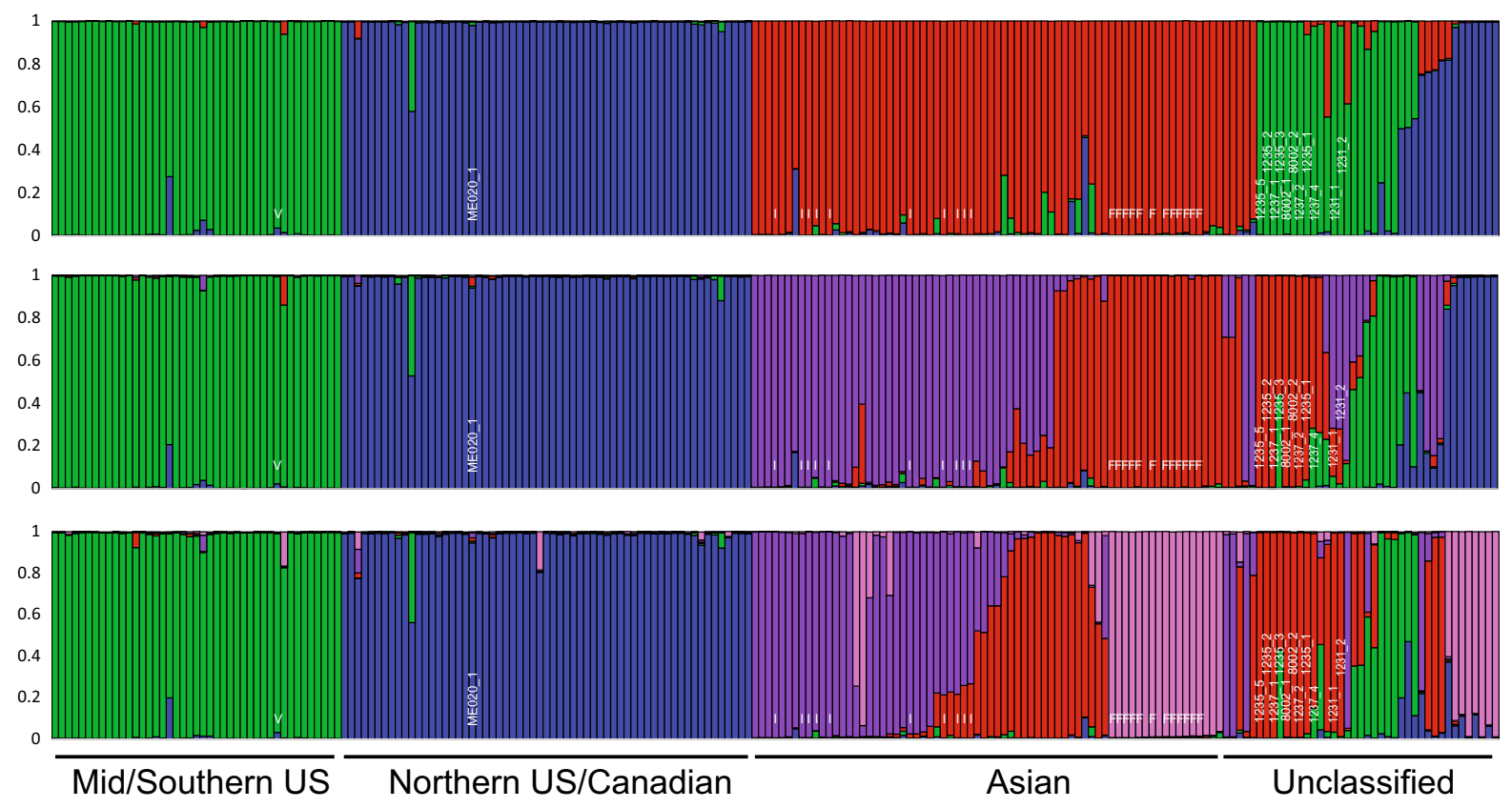

Fig. 1 Structure plots for $K=3, K=4$ and $K=5$. Genotypes represented in the plot are listed in the same order as in Online Resource 3. All genotypes are $S$. viridis except those indicated with 'V' (S. verticillata), 'I' (S. italica) and 'F' (S. faberi). Some

\section{Results}

Population structure

\section{Population structure analysis}

A total of 266 lines were genotyped with 11 SSR markers. The majority of the lines (226 lines) had no missing data, 34 lines had missing data for 1 SSR and 6 lines had missing data for 2 SSRs. After grouping lines with identical genotypes at all 11 loci, a total of $192 \mathrm{~S}$. viridis entries, $11 \mathrm{~S}$. faberi entries, $11 \mathrm{~S}$. italica entries and one $S$. verticillata entry were analyzed using STRUCTURE and InStruct. Lines with identical genotypes are given in Online Resource 2.

The $\Delta \mathrm{K}$ plot based on $\mathrm{LnP}(\mathrm{D})$ values from the STRUCTURE analysis indicated that the most likely number of populations (K) was 3. InStruct, however, indicated that, based on the deviance information criteria (DIC), the optimal value of K was 17. Because the accuracy of various methods for determining the optimal number of subpopulations is greatly decreased when using a small number of markers (Gao et al. 2011), we conducted both STRUCTURE and InStruct accessions discussed in the text are annotated by name. The 'unclassified' group consists of accessions that change subpopulation with varying $\mathrm{K}$. (Color figure online)

analyses with $\mathrm{K}$ varying from 3 to 6 , and then manually assessed the composition of subpopulations as $\mathrm{K}$ increased (Fig. 1; Online Resource 3). For the subgroup descriptions below, we only considered accessions that belonged to the same subpopulations as determined by both STRUCTURE and InStruct, and that did not change membership across the three main groups identified at $\mathrm{K}=3$ with increasing $\mathrm{K}$ value. At $\mathrm{K}=3$, the accessions largely separated into a Northern US/Canadian $S$. viridis group (latitudes above $46^{\circ} \mathrm{N}$ ), a Mid/Southern US S. viridis group which also comprised the $S$. verticillata accession (latitudes below $44^{\circ} \mathrm{N}$ ), and a predominantly Asian mixed group which contained $S$. viridis as well as the cultivated $S$. italica and the tetraploid wild species $S$. faberi. Subpopulation groupings largely corresponded with climatic zones (Online Resource 1). Increasing K to 4 placed all $S$. faberi accessions and a subset of the S. viridis accessions in a separate subgroup (Fig. 1). A further increase to $\mathrm{K}=5$ reduced the number of $S$. viridis accessions associated with $S$. faberi. The $S$. viridis accessions that split off from $S$. faberi, together with some other $S$. viridis accessions, formed a separate subpopulation at $\mathrm{K}=5$. Increasing $\mathrm{K}$ to 6 
divided the Northern US/Canadian group into 2 subgroups (Online Resource 3). Overall, 176 of the 215 unique genotypes had membership to the same subpopulation at $\mathrm{K}=3 \mathrm{using}$ both STRUCTURE and InStruct, and retained membership to those three overarching groups even when $\mathrm{K}$ was increased. The remaining 39 genotypes varied in their membership with varying $\mathrm{K}$ (Fig. 1; Online Resource 3). The change was largely unidirectional to the Asian group. For example, accessions 1231, 1235, 1237 and 8002 belonged to the Mid/Southern US S. viridis subpopulation at $\mathrm{K}=3$ and to the Asian subpopulation at $\mathrm{K}=4,5$ and 6 (Fig. 1; Online Resource 3). Of the lines that switched population groupings as $\mathrm{K}$ was modified, $24(62 \%)$ were solidly assigned to a particular group at $\mathrm{K}=3$ (membership value $\geq 95 \%$ ). Of these, $62 \%$ belonged to the Mid/Southern US group, $29 \%$ belonged to the Northern US/ Canadian group, and $8 \%$ belonged to the Asian group.

\section{Principal coordinates analysis}

The first three coordinates explained $19.64 \%$ of the variation, with the first coordinate explaining $9.62 \%$, the second coordinate $5.40 \%$ and the third coordinate $4.63 \%$. Color-coding of the accessions in the 2-dimensional PCoA plot showed a good correspondence between the population groups obtained from the PCoA and from the STRUCTURE/InStruct analyses (Fig. 2). Lines that changed membership with varying runs and/or $\mathrm{K}$ in the STRUCTURE/InStruct analyses largely grouped with the Asian accessions.

\section{Neighbor-joining tree}

Clustering of accessions in the neighbor joining tree generated with 11 SSR markers again was in good agreement with that obtained by the population structure and PCoA analyses (Fig. 3). To assess the robustness of the clustering, we generated distance matrices and trees based on subsets of 10 SSR markers and compared the tree topologies (Online Resource 4). Bar a few lines, the Mid/Southern accessions formed a single cluster in all trees. The Northern US/Canadian accessions largely grouped together in seven distance trees, and formed two clusters in four trees. The accessions belonging to the Asian subpopulation clustered to some extent, but these clusters were less

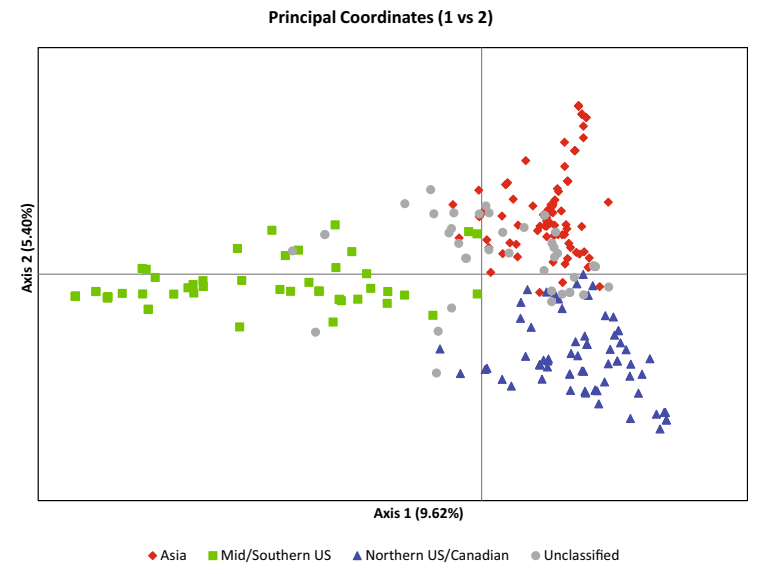

Fig. 2 Principal coordinates analysis. Accessions are color coded according to the subpopulation to which they had majority $(>50 \%)$ membership based on STRUCTURE and InStruct analyses (red diamonds Asian subpopulation; green squares Mid/Southern US subpopulation; blue triangles Northern US/Canadian subpopulation; gray circles unclassified). (Color figure online)

robust than the North American/Canadian and Mid/ Southern US clusters.

Overall genetic diversity

Genetic diversity is correlated with geographic distance

Significant positive correlations were found between the genetic distance and the geographic distance between accessions. This was true when all accessions were considered as well as for accessions within each of the three subpopulations. The correlation (Rxy value) was highest for accessions in the Asian subpopulation and lowest for accessions in the Northern US/Canadian subpopulation (Table 1).

\section{Genetic diversity by subpopulation}

All 11 SSR markers used showed polymorphisms in all three subpopulations, with the numbers of alleles varying from four (SSR p16 in the North American group) to 28 (SSR b101 in the Asian group) (Table 2). Both the mean number of alleles and effective number of alleles were lowest in the Mid/Southern US subpopulation $(7.7 ; 2.5)$ and highest in the Asian subpopulation (20.4; 10.6) (Table 2). Interestingly, in the Mid/Southern US subpopulation, a single allele was present at a 


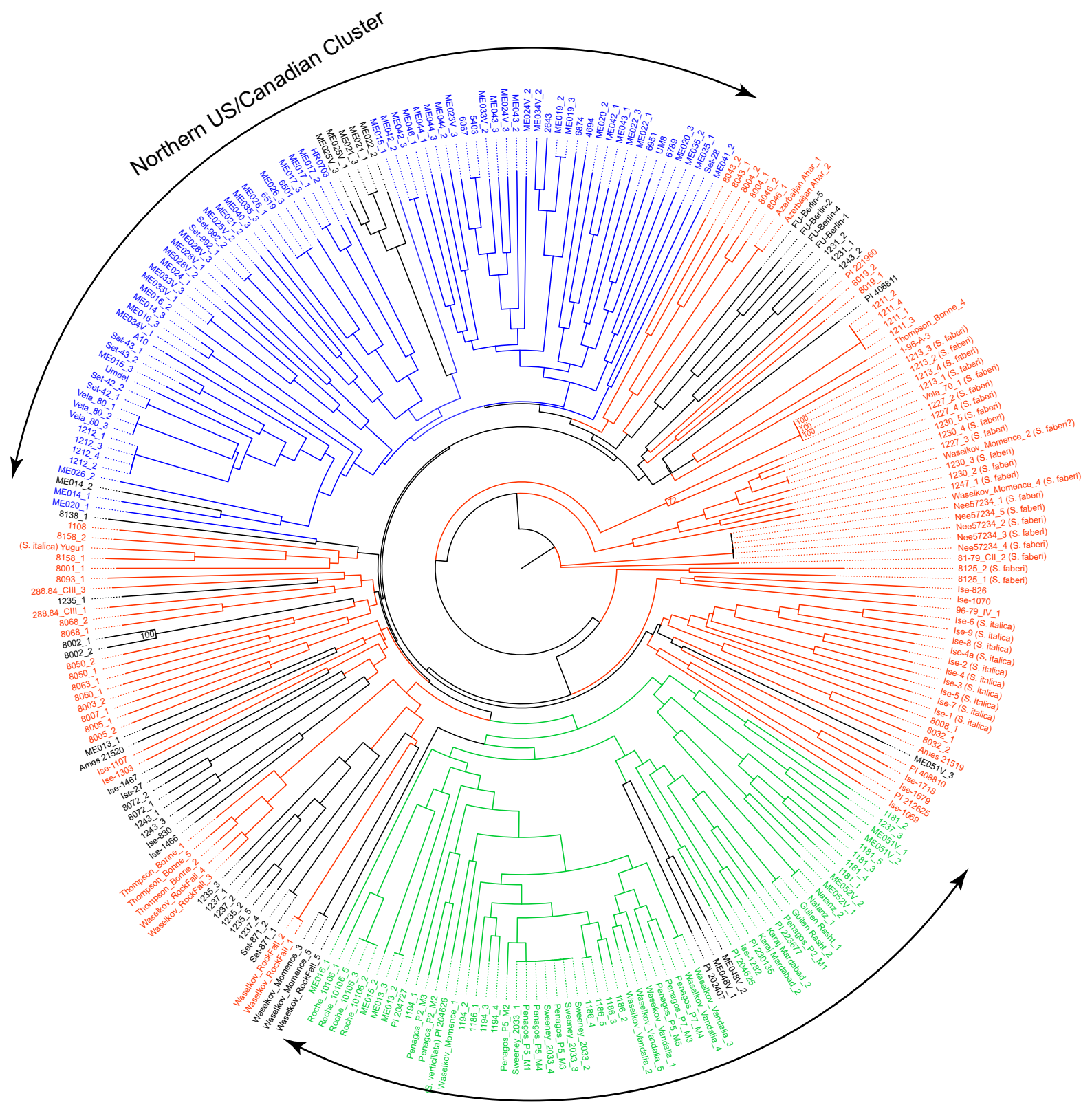

Mid/Southern US Cluster

Fig. 3 Neighbor joining tree. Accessions are color coded according to the subpopulation to which they had majority $(>50 \%)$ membership based on STRUCTURE and InStruct

frequency $>50 \%$ for eight of the 11 loci analyzed (Table 2; Online Resource 5). For six of these loci, the allele that was predominant in the Mid/Southern analyses (red Asian subpopulation; green Mid/Southern US subpopulation; blue Northern US/Canadian subpopulation; black unclassified). (Color figure online)

subpopulation was either absent or present at a frequency $<5 \%$ in both the Asian and Northern US/Canadian subpopulations. In the Northern US/Canadian 
Table 1 Correlation between genetic distance and geographic distance based on a Mantel test

\begin{tabular}{lll}
\hline Population & Rxy & P(rxy-rand $\geq$ rxy-data $)$ \\
\hline All & 0.353 & 0.001 \\
Asian & 0.562 & 0.001 \\
Mid/Southern US & 0.338 & 0.001 \\
Northern US/Canada & 0.267 & 0.001 \\
\hline
\end{tabular}

subpopulation, a predominant allele (frequency of $70.3 \%$ ) was found for a single SSR locus, p89, although this allele was also present at a frequency of $12.5 \%$ in the Asian subpopulation (Online Resource 5). In contrast, the highest frequency of any single allele in the Asian subpopulation was $30.9 \%$. Perhaps not surprisingly, $50 \%$ of all alleles in the Asian subpopulation were minor alleles (present in $\leq 5 \%$ of the individuals within a given subpopulation) compared to 11 and $27 \%$ of the alleles in the Mid/Southern US and Northern US/Canadian subpopulations, respectively.

On average, $71 \%$ of the variation was found between individuals within subpopulations, and $29 \%$ of the variation among subpopulations. The Asian subpopulation had the highest level of variation (expected heterozygosity ( $\mathrm{He}$ ) of $0.901 \pm 0.007$ ), followed by the Northern US/Canadian subpopulation
(He $=0.755 \pm 0.036)$ and the $\mathrm{Mid} /$ Southern US subpopulation $(\mathrm{He}=0.512 \pm 0.064)$.

\section{Discussion}

Genome relationships between $S$. viridis, S. faberi, $S$. verticillata and S. pumila

While the focus of this study was on $S$. viridis, we also collected $S$. faberi, which is morphologically highly similar to and hence can easily be confused with $S$. viridis, and $S$. pumila which often grows in sympatry with $S$. viridis. Several $S$. italica accessions, the domesticated form of $S$. viridis, were also included in our analysis. In addition, a single $S$. verticillata accession was obtained from GRIN. The SSR markers, which were developed against $S$. viridis sequence data (Jia et al. 2009) amplified equally well using S. viridis, S. italica, $S$. verticillata and $S$. faberi genomic DNA as a template. However, most primer sets failed to amplify or produced weak and/or complex patterns in S. pumila. This is in agreement with the results of phylogenetic analyses that place $S$. viridis, $S$. italica and one genome of the tetraploids $S$. faberi and $S$. verticillata into a single clade while $S$. pumila is more distantly related (Kellogg et al. 2009; Layton and

Table 2 Number of alleles (Na), effective alleles $(\mathrm{Ne})$ and heterozygosity (He) overall and per subpopulation

\begin{tabular}{|c|c|c|c|c|c|c|c|c|c|c|c|c|c|c|c|c|}
\hline \multirow[t]{2}{*}{ Marker } & \multirow{2}{*}{$\begin{array}{l}\text { Chromosomal } \\
\text { location }^{\text {a }}\end{array}$} & \multicolumn{4}{|c|}{ Asian } & \multicolumn{4}{|c|}{ Mid/Southern } & \multicolumn{4}{|c|}{ Northern US/Canada } & \multicolumn{3}{|l|}{ All } \\
\hline & & $\mathrm{Na}$ & $\mathrm{Ne}$ & $\mathrm{He}$ & Spe & $\mathrm{Na}$ & $\mathrm{Ne}$ & $\mathrm{He}$ & Spe & $\mathrm{Na}$ & $\mathrm{Ne}$ & $\mathrm{He}$ & Spe & $\mathrm{Na}$ & $\mathrm{Ne}$ & $\mathrm{He}$ \\
\hline p3 & $1(40.75 \mathrm{Mb})$ & 20 & 7.049 & 0.858 & - & 5 & 1.844 & 0.458 & 205 & 12 & 5.792 & 0.827 & - & 30 & 10.221 & 0.902 \\
\hline p29 & $7(31.74 \mathrm{Mb})$ & 24 & 13.501 & 0.926 & - & 7 & 2.854 & 0.650 & 180 & 10 & 5.432 & 0.816 & - & 37 & 13.518 & 0.927 \\
\hline b127 & $5(28.40 \mathrm{Mb})$ & 19 & 8.867 & 0.887 & - & 10 & 2.795 & 0.642 & 329 & 9 & 4.222 & 0.763 & - & 26 & 10.041 & 0.900 \\
\hline p88 & $1(29.83 \mathrm{Mb})$ & 21 & 11.306 & 0.912 & - & 7 & 1.781 & 0.438 & 217 & 9 & 4.261 & 0.765 & - & 29 & 11.368 & 0.912 \\
\hline b101 & $3(4.07 \mathrm{Mb})$ & 28 & 8.721 & 0.885 & - & 5 & 1.338 & 0.253 & 261 & 20 & 5.531 & 0.819 & - & 40 & 10.368 & 0.904 \\
\hline p95 & $9(12.28 \mathrm{Mb})$ & 21 & 12.117 & 0.917 & - & 6 & 1.532 & 0.347 & - & 11 & 4.859 & 0.794 & - & 24 & 6.475 & 0.846 \\
\hline p89 & $4(30.40 \mathrm{Mb})$ & 22 & 13.287 & 0.925 & - & 7 & 1.539 & 0.350 & 232 & 6 & 1.862 & 0.463 & - & 28 & 6.387 & 0.843 \\
\hline p16 & $1(32.95 \mathrm{Mb})$ & 15 & 8.591 & 0.884 & - & 6 & 1.267 & 0.211 & - & 4 & 2.530 & 0.605 & - & 17 & 4.976 & 0.801 \\
\hline b102 & $9(5.90 \mathrm{Mb})$ & 22 & 14.013 & 0.929 & - & 12 & 4. & 94 & - & 16 & 58 & 3 & - & 38 & 300 & 0.943 \\
\hline b166 & $9(14.11 \mathrm{Mb})$ & 19 & 11.620 & 0.914 & - & 12 & 4.658 & 0.785 & - & 16 & 7.027 & 0.858 & - & 27 & 13.679 & 0.927 \\
\hline b163 & $3(47.64 \mathrm{Mb})$ & 13 & 7.844 & 0.873 & - & 8 & 3.406 & 0.706 & - & 11 & 4.778 & 0.791 & - & 24 & 11.862 & 0.915 \\
\hline Mean & & 20 & 10.629 & 0.901 & & 8 & 2.533 & 0.512 & & 11 & 4.696 & 0.756 & & 29 & 10.609 & 0.893 \\
\hline
\end{tabular}

For each marker, the allele (in bp) that is present at a frequency of $>50 \%$ in one of the subpopulations and $<5 \%$ in both other subpopulations is indicated as specific alleles (Spe)

a The chromosomal location was obtained by conducting a Blastn search of the primer sequences listed in Jia et al. (2009) against the S. italica genome sequence (Bennetzen et al. 2012) 
Kellogg 2014). Our analysis of 30 S. pumila accessions with 11 SSR markers yielded no evidence of gene flow between $S$. pumila and $S$. viridis growing in sympatry.

Relationships between accessions based on population structure and neighbor-joining tree

Eighty-nine percent of the S. viridis lines (93\% of the accessions) from regions in the US covered by our analysis (Online Resource 1) can be grouped into two subpopulations, referred to as a Mid/Southern US group and a Northern US/Canadian group (Figs. 1, 2). Groupings obtained using STRUCTURE/InStruct aligned completely with the relationships revealed by a neighbor joining analysis except for one line, ME020_1, which was classified as belonging to the Northern US/Canadian subpopulation by STRUCTURE and InStruct, but grouped with Chinese accessions in the neighbor-joining tree (Fig. 3). ME020_1 carried rare alleles at three loci and also had missing data at two loci which may explain its odd placement in the neighbor joining tree. ME020_1 did, however, carry the 204 bp allele at locus p89, which is present in $69 \%$ of the lines belonging to the Northern US/Canadian subpopulation. Interestingly, removal of SSR b127 from the analysis clustered ME020_1 with the Northern US/Canadian subpopulation (Online Resource 4).

Accessions that belong to the Mid/Southern US subpopulation are found mainly below latitudes $44^{\circ} \mathrm{N}$ in Köppen's climate zone Cfa (warm temperate, fully humid, hot summer) (Kottek et al. 2006), while accessions belonging to the Northern US/Canadian subpopulation are found mainly above latitude $46^{\circ} \mathrm{N}$ in climate zone Dfb (snow climate, fully humid, warm summer). These results are in agreement with observations made by Wang et al. (1995) based on isozyme analysis of US $S$. viridis accessions and by Huang et al. (2014) based on close to 40,000 single nucleotide polymorphisms. The majority of the accessions from Iran and Turkey (climate zones Csa-warm temperate, dry hot summer; Csb-warm temperate, dry warm summer) grouped with the Mid/Southern US subpopulation suggesting that Mid/Southern US accessions may be derived from introductions from Southern Europe and/or the Middle East. The number of introductions with favorable allele combinations that gave rise to the Mid/Southern population may have been limited as indicated by the presence of predominant alleles at eight of the 11 SSR loci. The single $S$. verticillata line that was included in our analysis originated from Turkey and also grouped with the Mid/Southern US lines in both the population structure and neighbor joining analyses. The Western European accessions we analyzed (climate zone $\mathrm{Cfb}$-warm temperate, fully humid, warm summer) largely grouped with the Northern US/Canadian subpopulation, suggesting that introductions from Western Europe may have given rise to the Northern US and Canadian S. viridis populations. Because a predominant allele is found only at a single SSR locus, the Northern US/Canadian subpopulation probably originated from a larger number of introductions than the Mid/Southern subpopulation. The observed groupings likely reflect the differential adaptation of Turkish, Iranian and Mid/Southern US lines to dry or humid hot summers with $<15 \mathrm{~h}$ day lengths on one hand, and the Western European and Northern US/Canadian lines to humid and warm (but not hot) summers with $>15 \mathrm{~h}$ day lengths on the other hand. The SSR with the predominant allele in the Northern US/Canadian subpopulation is located on foxtail millet chromosome IV in a region that carries a flowering time QTL and may be associated with adaptation to Northern climates. The Mid/Southern US subpopulation has a different predominant allele at this locus. Differential adaptation to environmental conditions at different latitudes could be observed clearly when Canadian accessions were grown in the glasshouse in Georgia. Most accessions flowered very early, yielding mature plants that were very small and set little seed. This plant phenotype was very different from that observed when the plants grew in their native environment.

In contrast to the two largely North American subpopulations that formed distinct clusters in the neighbor-joining tree, lines belonging to the Asian subpopulation largely fell into three clusters (Fig. 3), but membership to the clusters varied depending on the software used to generate the distance matrices and trees, and on the subset of SSRs used in the analysis (Online Resource 4). This can likely be explained by the diversity of the accessions that formed the Asian subpopulation. No alleles were identified in the Asian subpopulation that were present in $50 \%$ or more of the accessions, and $50 \%$ of the alleles were minor alleles. This is in contrast to the Mid/Southern US population which had low genetic variation with predominant 
alleles at $72 \%$ of the SSR loci leading to stable clustering of accessions across different analyses.

In addition to $S$. viridis, our study included eight $S$. faberi accessions and $11 \mathrm{~S}$. italica accessions, all of which belonged to the Asian subpopulation. The $10 \mathrm{~S}$. italica accessions from India consistently clustered together and were more closely related to $S$. viridis accessions from India and Afghanistan than to Yugu1, a S. italica accession from China (Fig. 3). The cogrouping of $S$. italica with $S$. viridis from the same geographic region is consistent with previously published data (Le Thierry d'Ennequin et al. 2000) and indicates that gene flow exists between the two species. The $S$. faberi accessions also consistently clustered together. It had previously been suggested that $S$. faberi was introduced into the US from China (Rominger 1962). While membership of the S. faberi lines to the Asian subpopulation seems to support this, the fact that $S$. faberi accessions formed a separate cluster in the neighbor joining tree and that increasing $\mathrm{K}$ from 3 to 4 in the STRUCTURE and InStruct analyses resulted in the splitting off of the $S$. faberi accessions and some $S$. viridis lines from the Asian subpopulation indicates that this interpretation needs to be treated with caution. The $S$. viridis lines that grouped with $S$. faberi at $\mathrm{K}=4$ belonged to four accessions from the US, four accessions from China, one accession from Germany and one accession from Iran, which is too small a dataset to determine a country bias. In our collection of Setaria accessions, we had one accession (Waselkov_Momence) for which, of the five collected lines, one was classified as $S$. faberi based on glume size while the others were confirmed as $S$. viridis. Interestingly, one of the $S$. viridis lines (Waselkov_Momence 2) also fell into the S. faberi cluster in the neighbor-joining tree. Waselkov_Momence_2 carried alleles that were common in S. faberi at nine of the 11 loci. However, at two of the loci, the alleles in Waselkov_Momence 2 were absent from any of the $S$. faberi lines but were present in $S$. viridis, one at a low frequency in the Asian $(\sim 8 \%)$ and Northern US/Canadian subpopulation ( $\sim 3 \%)$, and the other at high $(\sim 80 \%)$ and moderate $(\sim 20 \%)$ frequencies in the Mid/Southern US subpopulation and Northern US/Canadian subpopulation, respectively. S. faberi is an allotetraploid with one genome donated by $S$. viridis, so it is not surprising that the two species should share alleles (Benabdelmouna et al. 2001; Layton and Kellogg 2014).
Although the diversity analysis was conducted with 11 SSRs only, the results of the population structure analyses were highly similar to those obtained by Huang et al. (2014) who analyzed a largely overlapping set of germplasm using $~ 40,000$ SNP markers obtained using genotyping-by-sequencing (GBS). Of the 112 lines that were in common between the two studies and that consistently grouped within the same subpopulation at $\mathrm{K}=3$ in the SSR study, all but two (98\%) were classified in the same subpopulation using SNPs and SSRs. For the purpose of comparison, lines were classified to the subpopulation in which they had $>50 \%$ membership. The GBS data, however, indicated a higher percentage of admixed lines ( $<90 \%$ membership to a single subpopulation) than the SSR analysis (33 vs. $6 \%$ ). Lines that were classified as admixed in the SSR analysis were also classified as admixed in the SNP analysis. The only exceptions were the lines Azerbaiyan Ahar and PI 221960 which were classified as admixed with a majority membership to the Northern US/Canadian subpopulation in the GBS study but belonged to the Asian subpopulation in the SSR analysis. These were the only two accessions in our study that originated from climate zone Dsb (snow climate with dry warm summers). When considering the 24 lines that were in common between the SNP and SSR studies, but did not have a consistent membership to a particular subpopulation using different software packages and/ or different $\mathrm{K}$ values based on the 11 SSRs, 54 and $79 \%$ of classifications agreed between the GBS data and the SSR results obtained at $\mathrm{K}=3$ and $\mathrm{K}=4$, respectively. At $K=4$, the Asian group largely split into two subgroups, and several of the lines that were classified as Mid/Southern US in the SSR analysis at $\mathrm{K}=3$ belonged to one of the two Asian subgroups at $\mathrm{K}=4$. The lines that differed in their classification between GBS SNP and SSR data were typically highly admixed (membership to a single subpopulation was $<60 \%)$.

\section{Conclusions}

Small numbers of SSR markers can be sufficient, depending on the species and genetic diversity present, to determine the overall population structure of germplasm collections. To solidly determine population groups, especially at low marker numbers, it can 
be helpful to assess the stability of line classifications at increasing $\mathrm{K}$ values. Lines that change membership may be more likely to have high levels of undetected admixture. Our analysis demonstrated that S. viridis lines in the US were likely introduced from Europe and/or the Middle East. The fact that Northern US and Canadian populations have a closer genetic relationship to $S$. viridis populations from Western Europe, and Mid/Southern US populations have a closer genetic relationship to $S$. viridis populations from Southern Europe and the Middle East suggests that $S$. viridis will only flourish if introduced to the climatic and/or photoperiod zones from which it originates and to which it is adapted.

Acknowledgments We thank D. Vela, K. Waselkov, J. Thompson, P. Sweeney, C. Roché, J. Penagos, M. Weigend, H. Beckie, T. Robert, M. Keshavarzi, A. Börner, USDA and ICRISAT for sharing of Setaria seed. This work was funded by National Science Foundation awards DEB-0952177 and DEB0952185 to KMD and EAK, respectively.

\section{Compliance with ethical standards}

Conflict of interest The authors declare that they have no conflict of interest.

Open Access This article is distributed under the terms of the Creative Commons Attribution 4.0 International License (http:// creativecommons.org/licenses/by/4.0/), which permits unrestricted use, distribution, and reproduction in any medium, provided you give appropriate credit to the original author(s) and the source, provide a link to the Creative Commons license, and indicate if changes were made.

\section{References}

Benabdelmouna A, Shi Y, Abirached-Darmency M, Darmency $\mathrm{H}$ (2001) Genomic in situ hybridization (GISH) discriminates between the $\mathrm{A}$ and the $\mathrm{B}$ genomes in diploid and tetraploid Setaria species. Genome 44:685-690

Bennetzen JL, Schmutz J, Wang H, Percifield R, Hawkins J, Pontaroli AC, Estep M, Feng L, Vaughn J, Grimwood J, Jenkins J, Barry K, Lindquist E, Hellsten U, Deshpande S, Wang X, Wu X, Mitros T, Triplett J, Yang X, Ye C-Y, Mauro-Herrera M, Wang L, Li P, Sharma M, Sharma R, Ronald PC, Panaud O, Kellogg EA, Brutnell T, Doust AN, Tuskan GA, Rokhsar D, Devos KM (2012) Reference genome sequence of the model plant Setaria. Nat Biotechnol 30:555-561

Blackshaw RE, Stobbe EH, Sturko ARW (1981) Effect of seeding dates and densities of green foxtail (Setaria viridis) on the growth and productivity of spring wheat (Triticum aestivum). Weed Sci 29:212-217
Brutnell TP, Wang L, Swartwood K, Goldschmidt A, Jackson D, Zhu XG, Kellogg E, Van Eck J (2010) Setaria viridis: a model for C-4 photosynthesis. Plant Cell 22:2537-2544

Darmency H (2005) Incestuous relations of foxtail millet (Setaria italica) with its parents and cousins. Crop ferality and volunteerism. CRC Press, Boca Raton, pp 81-96

Douglas BJ, Gordon TA, Morrison IN, Maw MG (1985) The biology of Canadian weeds. 70. Setaria viridis (L.) Beauv. Can J Plant Sci 65:669-690

Doust AN, Kellogg EA, Devos KM, Bennetzen JL (2009) Foxtail millet: a sequence-driven grass model system. Plant Physiol 149:137-141

Doyle JJ, Doyle JL (1987) A rapid DNA isolation procedure for small quantities of fresh leaf tissue. Phytochem Bull 19:11-15

Evanno G, Regnaut S, Goudet J (2005) Detecting the number of clusters of individuals using the software structure: a simulation study. Mol Ecol 14:2611-2620

Fairbrothers DE (1959) Morphological variation of Setaria faberii and S. viridis. Brittonia 11:44-48

Felsenstein J (1993) PHYLIP (Phylogeny Inference Package) version $3.5 \mathrm{c}$. Distributed by the author. Department of Genetics, University of Washington, Seattle

Forcella F, Harvey SJ (1983) Relative abundance in an alien weed flora. Oecologia 59:292-295

Gao H, Williamson S, Bustamante CD (2007) A Markov Chain Monte Carlo approach for joint inference of population structure and inbreeding rates from multilocus genotype data. Genetics 176:1635-1651

Gao H, Bryc K, Bustamante CD (2011) On identifying the optimal number of population clusters via the deviance information criterion. PLoS ONE 6:e21014

Groh H, Frankton C (1949) Canadian weed survey. 7th report. Canada Department of Agriculture, Ottawa, p 144

Huang P, Feldman M, Schroder S, Bahri BA, Diao X, Zhi H, Estep M, Baxter I, Devos KM, Kellogg EA (2014) Population genetics of Setaria viridis, a new model system. Mol Ecol 20:4912-4925

Jia X, Zhang Z, Liu Y, Zhang C, Shi Y, Song Y, Wang T, Li Y (2009) Development and genetic mapping of SSR markers in foxtail millet [Setaria italica (L.) P. Beauv.]. Theor Appl Genet 118:821-829

Jia G, Shi S, Wang C, Niu Z, Chai Y, Zhi H, Diao X (2013) Molecular diversity and population structure of Chinese green foxtail [Setaria viridis (L.) Beauv.] revealed by microsatellite analysis. J Exp Bot 64:3645-3656

Jiang H, Barbier H, Brutnell T (2013) Methods for performing crosses in Setaria viridis, a new model system for the grasses. J Vis Exp 80:e50527

Kellogg EA, Aliscioni SS, Morrone O, Pensiero J, Zuloaga F (2009) A phylogeny of Setaria (Poaceae, Panicoideae, Paniceae) and related genera, based on the chloroplast gene $n d h F$. Int J Plant Sci 170:117-131

Knake EL (1990) Giant foxtail: Setaria faberi Herrm. Bulletin of the University of Illinois at Urbana-Champaign, College of Agriculture, Agricultural Experiment Station 803

Kottek M, Grieser J, Beck C, Rudolf B, Rubel F (2006) World map of the Köppen-Geiger climate classification updated. Meteorol Z 15:259-263

Layton DJ, Kellogg EA (2014) Morphological, phylogenetic, and ecological diversity of the new model species Setaria 
viridis (Poaceae: Paniceae) and its close relatives. Am J Bot 101:539-557

Le Thierry d'Ennequin M, Panaud O, Toupance B, Sarr A (2000) Assessment of genetic relationships between Setaria italica and its wild relative $S$. viridis using AFLP markers. Theor Appl Genet 100:1061-1066

Li P, Brutnell T (2011) Setaria viridis and Setaria italica, model genetic systems for the Panicoid grasses. J Exp Bot 62:3031-3037

Li CH, Pao WK, Li HW (1942) Interspecific crosses in Setaria. II. Cytological studies of interspecific hybrids involving: 1 . S. faberii and 2. a three way cross, $\mathrm{F}_{2}$ of $S$. italica $\times S$. viridis and $S$. faberii. J Hered 33:351-355

Liu K, Muse SV (2005) PowerMarker: an integrated analysis environment for genetic marker analysis. Bioinformatics 21:2128-2129

Manson JM (1932) Weed survey of the Prairie Provinces. Dominion of Canada, p 34

Mantel N (1967) The detection of disease clustering and a generalized regression approach. Cancer Res 27:209-220

Naciri Y, Darmency H, Belliard J, Dessaint F, Pernès J (1992) Breeding strategy in foxtail millet, Setaria italica (L.P. Beauv.), following interspecific hybridization. Euphytica 60:97-103

Peakall R, Smouse PE (2012) GenAlEx 6.5: genetic analysis in Excel. Population genetic software for teaching and research-an update. Bioinformatics 28:2537-2539

Peterson DE, Nalewaja JD (1992) Green foxtail (Setaria viridis) competition with spring wheat (Triticum aestivum). Weed Technol 6:291-296

Pritchard JK, Stephens M, Donnelly P (2000) Inference of population structure using multilocus genotype data. Genetics 155:945-959
Qie L, Jia G, Zhang W, Schnable J, Shang Z, Li W, Liu B, Li M, Chai Y, Zhi H, Diao X (2014) Mapping of quantitative trait locus (QTLs) that contribute to germination and early seedling drought tolerance in the interspecific cross Setaria italica $\times$ Setaria viridis. PLoS ONE 9:e101868

Rominger JM (1962) Taxonomy of Setaria (Graminaceae) in North America. University of Illinois Press, Urbana

Till-Bottraud I, Reboud X, Brabant P, Lefranc M, Rherissi B, Vedel F, Darmency H (1992) Outcrossing and hybridization in wild and cultivated foxtail millets: consequences for the release of transgenic crops. Theor Appl Genet 83:940-946

Wang RL, Wendel JF, Dekker JH (1995) Weedy adaptation in Setaria spp.1. Isozyme analysis of genetic diversity and population genetic structure in Setaria viridis. Am J Bot 82:308-317

Wang T, Fleury A, Ma J, Darmency H (1996) Genetic control fo dinitroaniline resistance in foxtail millet (Setaria italica). J Hered 87:423-426

Willweber-Kishimoto E (1962) Interspecific relationships in the genus Setaria. Control Biol Kyoto Univ 14:1-41

Zhang G, Liu X, Quan Z, Cheng S, Xu X, Pan S, Xie M, Zeng P, Yue Z, Wang W, Tao Y, Bian C, Han C, Xia Q, Peng X, Cao R, Yang X, Zhan D, Hu J, Zhang Y, Li H, Li N, Wang J, Wang C, Wang R, Guo T, Cai Y, Liu C, Xiang H, Shi Q, Huang P, Chen Q, Li Y, Wang J, Zhao Z, Wang J (2012) Genome sequence of foxtail millet (Setaria italica) provides insights into grass evolution and biofuel potential. Nat Biotechnol 30:549-554 